【生物工程／Bioengineering】

\title{
小鼠纹状体与视网膜的比较转录组学分析
}

须周恒 ${ }^{1}$ ，朱营利 ${ }^{1}$ ，孙孟菲 ${ }^{1}$ ，魏志远 ${ }^{1}$ ，潘佳琪 ${ }^{1}$ ，潘旭斌 ${ }^{2}$, 陈建欢 ${ }^{1}$

1 ）江南大学无锡医学院, 江苏无锡 $214122 ； 2$ ）江南大学附属医院（无锡市第四人民医院）, 江苏无锡 214062

摘要：已发现以纹状体多巴胺减少为主要病变之一的帕金森综合症 (Parkinson's disease, PD), 可能 伴有视网膜病理改变. 通过分析比较小鼠纹状体及视网膜在转录组水平的基因表达谱异同, 探讨 PD 相关 视网膜病理改变的潜在分子基础. 对正常成年 C57BL/6 小鼠的纹状体和视网膜进行核糖体核糖核酸 (ribosomal ribonucleic acid, rRNA) 减除法建库、转录组测序和生物信息学分析，比较两者基因表达谱的异 同，分析差异基因所富集的基因功能和信号通路. 重点比较两者 PD 信号通路基因表达的异同，并构建相关 基因互作网络. 比较转录组学分析发现两者显著差异表达的基因共 2478 个. 其中, 1579 个基因在纹状体 中呈高表达; 899 个基因在视网膜中呈高表达. 在纹状体中，高表达基因富集的功能和通路主要与信号转 导及膜转运相关; 而在视网膜中，则表现为与转录和光转导相关. PD 信号通路有 11 个基因在纹状体高表 达，而在视网膜呈现显著低表达，该通路中其他大多数基因则在两种组织间有相似的表达模式. 研究结果 揭示了小鼠纹状体和视网膜在转录组水平上的基因表达谱的异同，并探索了与 PD 相关视网膜病变的潜在 分子基础.

关键词：神经生物学; 帕金森病; 二代测序; 转录组; 纹状体; 视网膜; 生物信息学; 基因互作网络 中图分类号：Q819; Q756 文献标志码：A doi : 10.3724/SP. J. 1249.2020.01017

\section{Comparative transcriptomic analysis of mouse striatum and retina}

\section{XU Zhouheng ${ }^{1}$, ZHU Yingli $^{1}$, SUN Mengfei ${ }^{1}$, WEI Zhiyuan $^{1}$, PAN Jiaqi ${ }^{1}$, PAN Xubin ${ }^{2}$, CHEN Jianhuan ${ }^{1}$}

1) Wuxi School of Medicine, Jiangnan University, Wuxi 214122, Jiangsu Province, P. R. China

2) Affiliated Hospital of Jiangnan University ( Wuxi 4th People's Hospital), Wuxi 214062, Jiangsu Province, P. R. China

\begin{abstract}
Parkinson's disease (PD), with decreased dopamine in the striatum as one of the major lesions, may be accompanied by retinopathy. By analyzing and comparing the gene expression patterns in the mouse striatum and retina at the transcriptome level, the potential molecular basis of PD-related retinopathy was explored. From normal adult C57BL/6 mice striatum and retinaribonucleic acid, library was constructed using ribosomal ribonucleic acid (rRNA) depletion, and sequenced on the Illumina Hiseq 4000 platform. After bioinformatic analysis of sequencing data, the expression profiles of the striatum and retina were compared, and differentially expressed genes were identified, followed by gene ontology ( GO ) and Kyoto encyclopedia of gene and genomes (KEGG) pathway analysis. Andgene networks were constructed from the differentially expressed genes. Results showed that a total of 2478 genes had significantly different expressions, among which 1579 and 899 genes were highly expressed in the
\end{abstract}

Received: 2019-01-22; Accepted: 2019-04-05

Foundation: National Natural Science Foundation of China (31671311)

Corresponding author: Professor CHEN Jianhuan. E-mail: cjh_bio@ hotmail.com

Citation: XU Zhouheng, ZHU Yingli, SUN Mengfei, et al. Comparative transcriptomic analysis of mouse striatum and retina [J]. Journal of Shenzhen University Science and Engineering, 2020, 37(1) : 17-24. (in Chinese) 
striatum and retina respectively. GO and KEGG analysis showed that genes highly expressed in the striatum were mainly enriched in signal transduction and membrane transport, while those highly expressed in the retina were associated with transcription and phototransduction. 11 genes in the PD pathway among the highly expressed genes in the striatum, showed significantly lower expression in the retina, while most of the other genes in the pathway had similar expression patterns between the two tissues. Our studythus demonstrated the similarities and differences in gene expression profiles between the mouse striatum and retina, and provided useful evidence tobetter understand the molecular basis of retinopathy in PD.

Key words : neurobiology; Parkinson's disease (PD) ; next-generation sequencing; transcriptome ; striatum ; retina ; bioinformatics; gene interaction network

帕金森病 (Parkinson's disease, PD) 是一种严重 的神经退行性疾病, 病变表现为中脑黑质多巴胺能 神经元退行性死亡, 以引起纹状体多巴胺含量显著 减少, 继而导致肌张力异常. 主要症状表现为以静 止性震颤、肌僵直、运动迟缓和姿势平衡障碍为主 的运动异常和以抑郁、便秘和睡眠障碍为主的非运 动症状 ${ }^{[1]}$. 纹状体由豆状核与尾状核组成, 是基底 神经节重要的组成部分. 豆状核由苍白球与壳核组 成. 纹状体中尾状核、壳核、苍白球与丘脑底核、 黑质在结构与功能上是紧密相联系的, 其中苍白球 是纤维联系的中心. 基底神经节有重要的运动调节 功能, 它与随意运动的产生和稳定、肌紧张的调节 和本体感受传人冲动信息的处理都有关系. 纹状体 因其重要功能在 PD 病理过程中扮演重要角色.

视网膜常被认为是相对独立于大脑的神经系 统. 但视网膜和视神经与大脑有着相同的胚胎起 源, 且有类似的血管形成、自我调节血流和血液组织屏障功能的模式. 作为中枢神经系统 (central nervous system, CNS) 的延伸, 视网膜具有的血-眼 屏障 (blood-retinal barrier, BRB), 其在结构上类似 于血脑屏障 (blood-brain barrier, BBB) 的组分和机 制, 具有特定的免疫豁免权. 近年来, 许多中枢神 经系统疾病被发现常伴有视觉系统的受累 ${ }^{[2-5]}$. RAMIREZ ${ }^{[6]}$ 指出, 视网膜病变可以是某些神经退行 性疾病, 如 PD 和阿尔兹海默症的一种临床表型, 有可能作为早期诊断的一个检测指标. 视觉幻觉 (visual hallucination, $\mathrm{VH}$ ) 是 $\mathrm{PD}$ 的一个特定症状, 且 PD 患者中多见视网膜多巴胺含量下降和中央凹 视力功能障碍 ${ }^{[7]}$. 而来自中心和外周途径的视觉信 息处理缺陷被认为是 PD 中 VH 的病理生理学机 制 ${ }^{[8]}$. 但是, PD 中视网膜受累的分子机制目前仍 然不明确.

许多基因被证明与 PD 相关, 其中， $\alpha$-突触核 蛋白 ( $\alpha$-synuclein, SNCA) 被认为参与多巴胺释放和
转运的调节, 诱导微管相关蛋白 tau 的纤维化, 并 通过抑制 p53 表达和促调亡基因的反式激活导致非 多巴胺能神经元发挥神经保护表型, 导致 caspase-3 活化减少 ${ }^{[23]}$, 其突变常常引起 PD 的发生.

比较转录组学, 是研究比较不同生物学组织或 样本之间的细胞/组织在转录组水平的异同点. 转 录组学被广泛应用于包括 PD 在内的神经系统疾病 的研究中 ${ }^{[9-13]}$. 但到目前为止, 尚未见对视网膜与 脑组织的基因表达谱差异进行比较, 以及挖掘其与 $\mathrm{PD}$ 相关生物学意义的有关报道. 本研究利用转录 组测序和生物信息学分析的方法对正常小鼠视网膜 及纹状体内表达差异的基因进行了分析, 对 PD 与 视网膜改变关系的重要分子基础进行探讨.

\section{1 材料与方法}

\section{1 动物组织的采集}

实验采用 3 只 12 周龄雄性 C57/BL6 小鼠, 获 得了江南大学动物伦理委员会的许可, 采用吸人式 麻醉剂异氟烷对小鼠进行麻醉后, 通过心脏灌注法 对小鼠处死 (小鼠的处死及组织取材方法请扫描论 文末页右下角二维码查看), 针对纹状体和左眼视 网膜进行组织取材料, 新鲜组织离体后迅速切成小 块并置于 Trizol 溶液中, 待组织块完全溶解后直接 进行总核糖核酸 (ribonucleicacid, RNA) 的提取. 将 3 个视网膜样本分别编号为 $R_{1} 、 R_{2}$ 和 $R_{3}, 3$ 个纹 状体样本分别编号为 $\mathrm{S}_{1} 、 \mathrm{~S}_{2}$ 和 $\mathrm{S}_{3}$.

\section{2 组织总 RNA 的提取}

利用酚-氯仿法对所采集的小鼠的纹状体和视 网膜组织进行总 RNA 的提取.

\subsection{RNA 测序}

所提取的总 RNA 使用质量浓度为 $10 \mathrm{~g} / \mathrm{L}$ 的琼 脂糖电泳进行降解与污染的检测, 并用 kaiaoK $550{ }^{\circledR}$ 分光光度计测纯度. RNA 的完整性和浓度用 
RNA Nano 6000 assay 试剂盒在 Bioanalyzer 2100 上 进行测定. 按试剂盒说明书, 使用 NEBNext ${ }^{\circledR}$ UltraTM Directional RNA library Prep kit 以核糖体 RNA 减除 法构建文库，并使用 $\mathrm{Qubit}^{\circledR}$ RNA assay kit 和 Qubit $^{\circledR} 2.0$ 进行文库质检. 所得文库在 Illumina Hiseq 4000 平台上使用 150 碱基对 (base pair, bp)进 行双端测序. 每个样本的测序量均不低于 12 Gbase.

\section{4 读段比较及差异表达基因的分析}

所得读段使用 Fastqc 进行质量评估. 将评估合 格读段比较到小鼠的全基因组序列（UCSC 版本 mm10）上. 基因的表达分析使用 FeatureCounts 进 行计数分析, 用 Bioconductor 的 edgeR 语言包进行 差异表达基因 (differentially expressed gene, DEG) 分析. 为保证得到高度可信的 DEG, 差异倍数 (flod change, FC) 的对数值 ( lb FC) ${ }^{[2]}$ 大于 2 , 较正后的 假阳性发现率 (false discovery rate, FDR) 小于 0.05 的基因，认为是带分析的候选 DEG.

\section{5 差异表达基因的组成分析}

利用 $\mathrm{R}$ 统计语言的 Bioconducter 软件包 pheatmap 绘制 DEG 的热图. 利用 DEG 数据和 ggplot2 软 件包中的 autoplot 函数绘制主成分分析图.

\section{6 差异表达基因的功能及通路富集分析}

利用 DAVID（版本 6.8 ) 生物信息学网站 (http:// david. ncifcrf. gov) 对纹状体和视网膜组织 的 DEG 进行功能富集 (gene ontology, GO) 和 Kyoto encyclopedia of genes and genomes (KEGG) 信号通路 富集分析 ${ }^{[14-15]}$.

\section{7 基因网络图分析}

利用 cytoscape (版本 3.7.1) 中的 STRING 软件 包( https://string-db. org) 对相关的 DEG 进行基因间 相互作用分析，并绘制网络图.

\section{2 结果与分析}

\section{1 小鼠纹状体与视网膜转录组差异表达比较}

对两组小鼠组织中的 DEG 分析. 结果表明, 有 2478 个基因出现了表达差异（FDR $<0.05$, $\mathrm{lb} \mathrm{FC}>2$ 或 $\mathrm{lb} \mathrm{FC}<-2)$. 其中, 899 个基因在纹状 体中相对于视网膜呈现低表达，有 1579 个基因在 纹状体中相对于视网膜呈现高表达, 由此构建纹状 体-视网膜基因差异表达热图如图 1 (a). 完整视网 膜及纹状体间差异表达基因的表达差异热图和数据 请扫描论文末页右下角二维码查看图 S1 和表 S1. 由图 1 和表 $\mathrm{S} 1$ 可见, 纹状体与视网膜的基因表达
谱有着较大的差别, 而主成分分析结果也可以看出 在组内差异不大的情况下, 两组样品可明显地聚为 两类. 由图 $1(b)$ 可见, DEG 主成分分析中的 PC1 对结果的解释度达到 $65.9 \%$.

\section{2 小鼠脑纹状体与视网膜差异表达基因的 GO 分析}

利用 DAVID 数据库分析纹状体-视网膜差异表 达的基因, 分别对纹状体内显著高表达及低表达的 基因进行 $\mathrm{GO}$ 分析, 显示纹状体内高表达的基因参 与的生物过程 (biological process, BP) 显著性最强 的 7 个聚类 (term) 分别为: 细胞通讯、信号通路、 定位、突触信号、信号转导、跨突触信号传导和化 学突触传递等. 参与的细胞组成 (cell component, $\mathrm{CC}$ ) 显著性最强的 7 个 term 分别为: 细胞外周、 膜、质膜、膜部分、神经元部分、质膜部分和膜的 内在组成部分. 参与的分子功能 (molecular function, MF) 分析显著性最强的 7 个 term 分别为: 蛋 白质结合、信号传感器活动、离子跨膜转运蛋白活 性、跨膜转运蛋白活性、无机分子实体跨膜转运蛋 白活性、分子功能调节剂和跨膜信号传导受体活 性，见图 2. 完整纹状体高表达基因的 GO 分析结 果请扫描论文末页右下角二维码见表 S2. 而视网膜 内高表达基因的 BP term 显著性前 7 名分别为: 核 酸代谢过程、RNA 代谢过程、调节 RNA 代谢过程、 调节含核碱基的化合物代谢过程、调节基因表达、 含核碱基的化合物代谢过程和 RNA 生物合成过程. 显著性前 7 位的 CC term 则为: 核、核质、核腔、 核部分、核质部分、感光细胞纤毛和感光器外段. MF 前 7 位的 term 分别为: DNA 结合、核酸结合、 转录调节活性、DNA 结合转录因子活性、序列特异 性 DNA 结合，以及双链 DNA 结合和调节区核酸结 合，见图 3. 完整视网膜高表达基因的 GO 分析结 果请扫描论文末页右下角二维码见表 S3. 纹状体内 更倾向于膜表面信号转导相关基因的高表达，而在 视网膜内, 则是与其功能有关的视觉感受器相关基 因表达更为活跃.

\section{3 小鼠脑纹状体与视网膜差异表达基因的 KEGG 通路分析}

将纹状体与视网膜差异表达的基因进行 KEGG 通路分析, 结果显示, 纹状体内高表达的基因主要 富集在核糖体、阿尔茨海默病、氧化磷酸化、帕金 森综合症、钙信号通路、亨廷顿病及神经活性配 体-受体相互作用通路上，见图 4. 完整纹状体高表 达 KEGG 分析结果请扫描论文末页右下角二维码见 


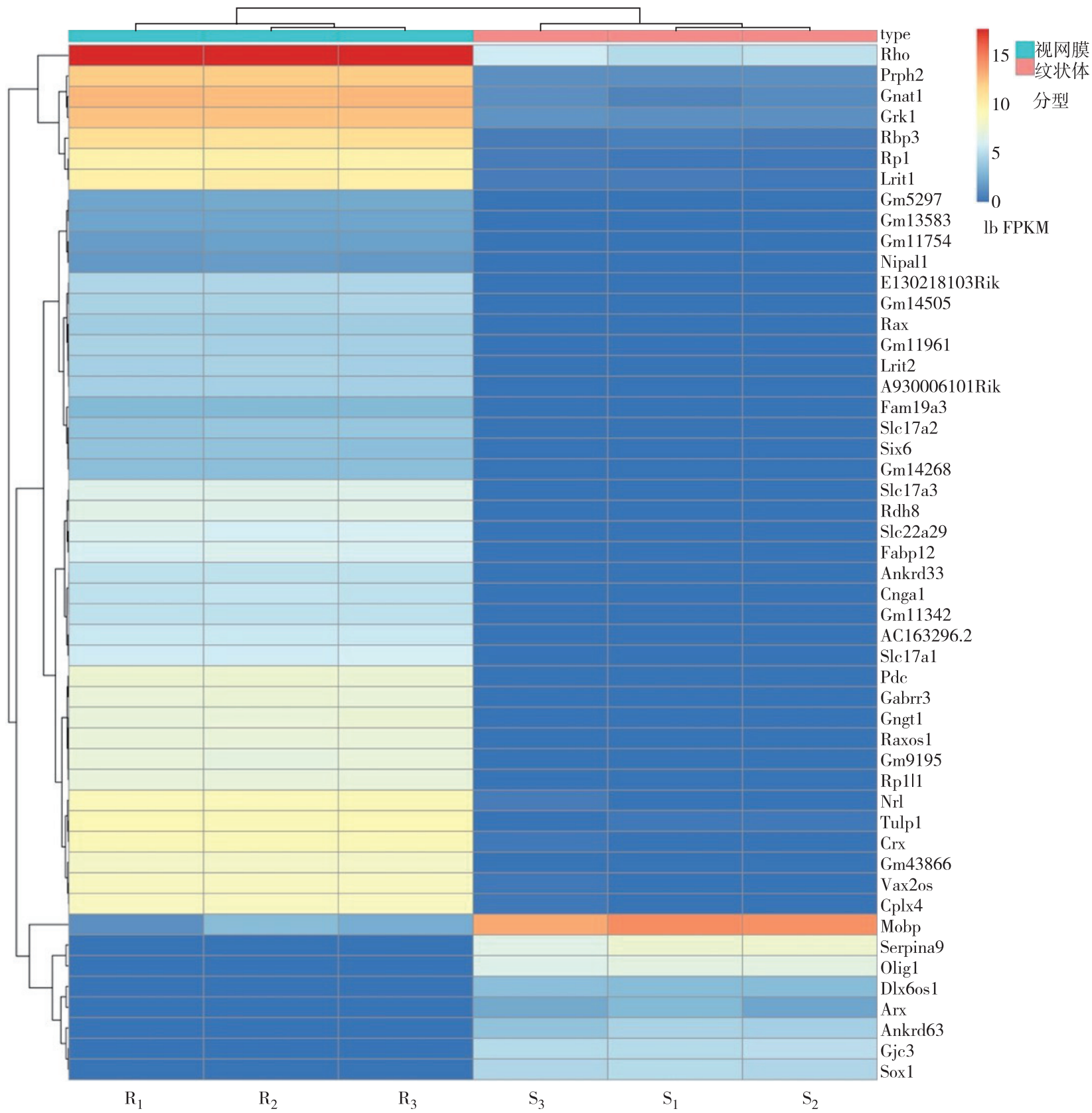

(a ) 两种组织间DEG $\mathrm{lbFC}$ 前50个基因的表达差异热图

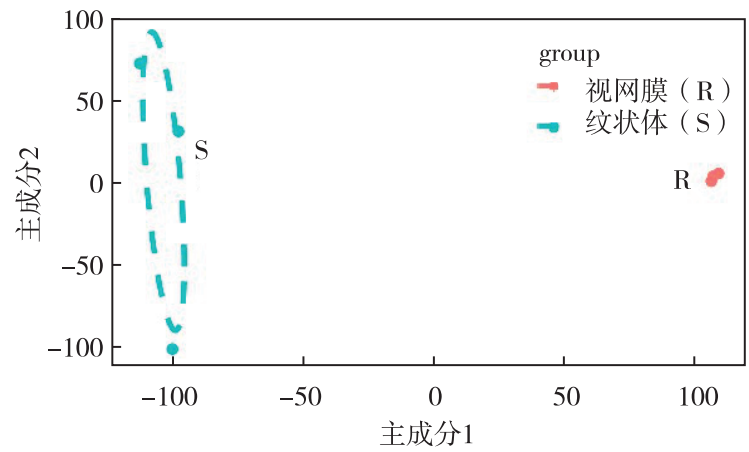

(b) 纹状体和视网膜

图 1 小鼠脑纹状体与视网膜转录组水平的差异表达热图

Fig. 1 (Color online) Differentially expressed genes between mouse striatum and retina 


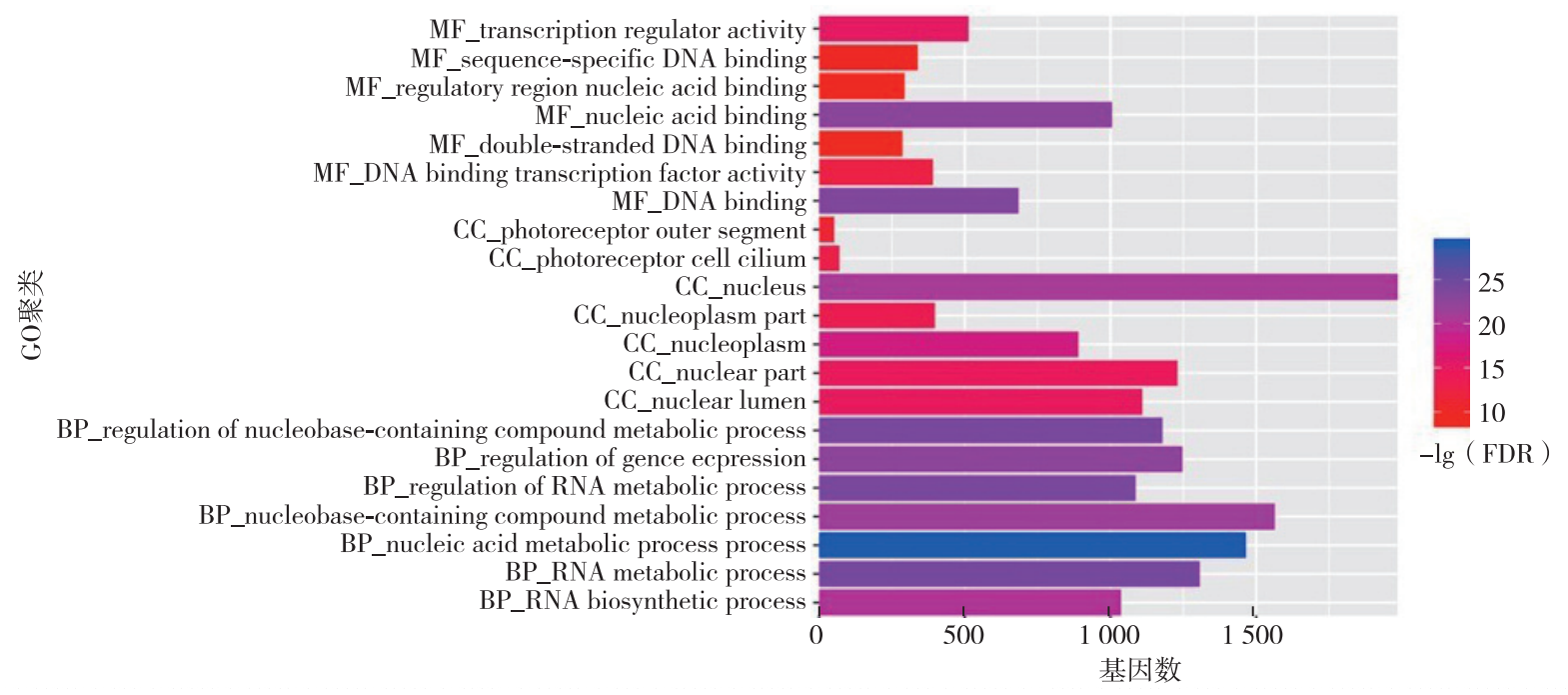

图 2 小鼠脑纹状体比对视网膜高表达差异基因的 GO 分析

Fig. 2 (Color online) Enriched genes with significantly higher expression in mouse striatum compared with those in the retina by $\mathrm{GO}$ analysis

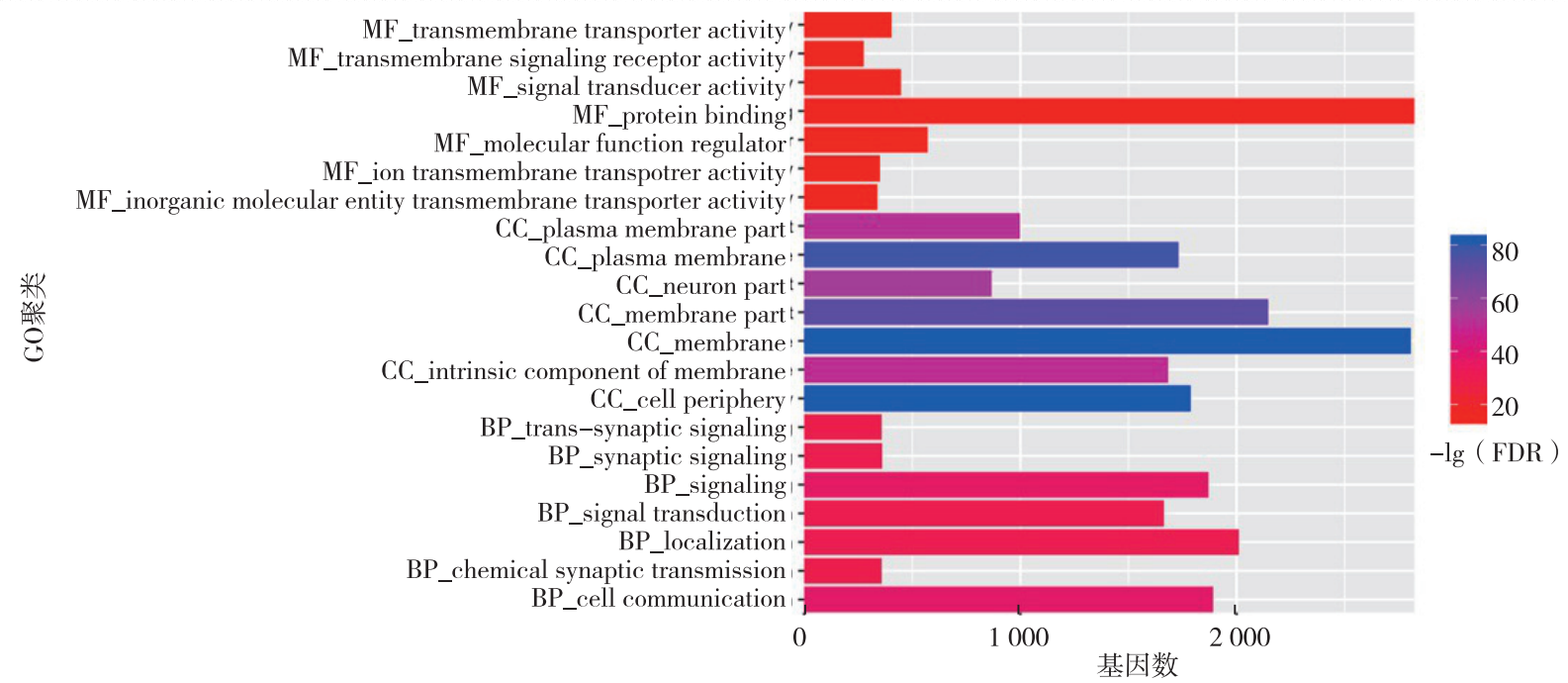

图 3 小鼠脑纹状体比对视网膜低表达差异基因的功能富集分析

Fig. 3 (Color online) Enriched genes with significantly lower expression in mouse striatum compared with those in the retina by $\mathrm{GO}$ analysis

表 S4. 而视网膜高表达的基因基因富集通路主要有 光转换、RNA 降解、剪接、错配修复、基础转录因 子、维生素消化吸收和细胞周期等，见图 5.（完 整视网膜内高表达基因 KEGG 分析结果请扫描论文 末页右下角二维码见表 S5). KEGG 通路分析结果 与观察到的 GO 分析的结果相互印证.

\subsection{PD 通路相关基因在纹状体和视网膜中的表达}

因小鼠脑纹状体高表达基因的 KEGG 数据分析 显示了这些基因明显地在帕金森病通路上富集，利 用 STRING 数据库的基因互作数据, 对鼠纹状体在 该通路中高表达的差异基因构建了基因互作网络, 见图 6. 同时, 针对所有 PD 通路基因在正常小鼠
脑纹状体和视网膜两种组织中的表达情况进行比 较. 结果发现, 共有 129 个 PD 通路基因被检测到 有不同程度的表达, 其中, 有 12 个基因 $|\mathrm{lb} \mathrm{FC}|>$ 2, 11 个在纹状体中高表达, 包括: adenosine A2a receptor (Adora2a)、 adenylate cyclase 5 (Adcy5)、 dopamine receptor D2 ( Drd2)、 guanine nucleotide binding protein alpha inhibiting 1 (Gnai1)、guanine nucleotide binding protein subunit alpha L (Gnal)、 leucine-rich repeat kinase $2(\operatorname{Lrrk} 2)$, septin 5 ( Sept5 )、 synuclein alpha interacting protein (Sncaip)、 synuclein alpha ( Snca)、ubiquinol-cytochrome c reductase、 complex III subunit XI (Uqcr11) 
和 ubiquitin carboxy-terminal hydrolase L1 (Uchl1). 有意思的是, 另一个差异基因 cytochrome c oxidase subunit IV isoform 2 (Cox4i2) 却是在视网膜高表达,

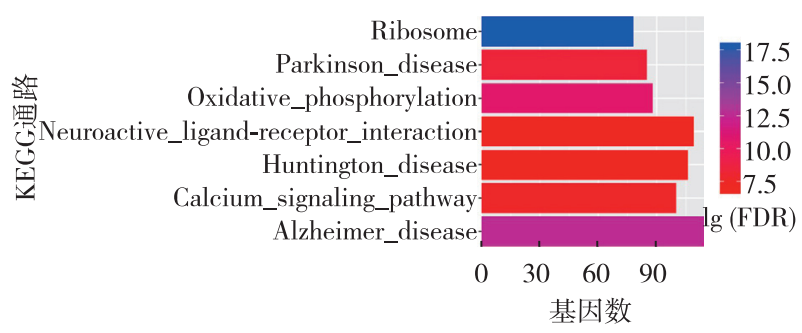

图 4 小鼠脑纹状体比对视网膜高表达 差异基因的 KEGG 通路分析

Fig. 4 (Color online) Pathways enriched genes with significantly higher expression in mouse striatum compared with those in the retina by KEGG analysis
见图 7. 尽管有该部分基因存在显著表达差异, 两 种组织表达的 PD 通路中的其余 117 个基因表达水 平却比较接近，呈现较为相似的表达模式.

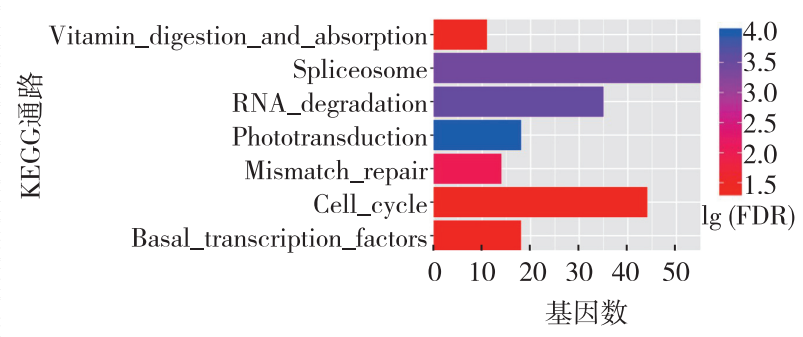

图 5 小鼠脑纹状体比对视网膜低表达 差异基因的 KEGG 通路分析

Fig. 5 (Color online) Pathways enriched genes with significantly lower expression in mouse striatum compared with those in the retina by KEGG analysis

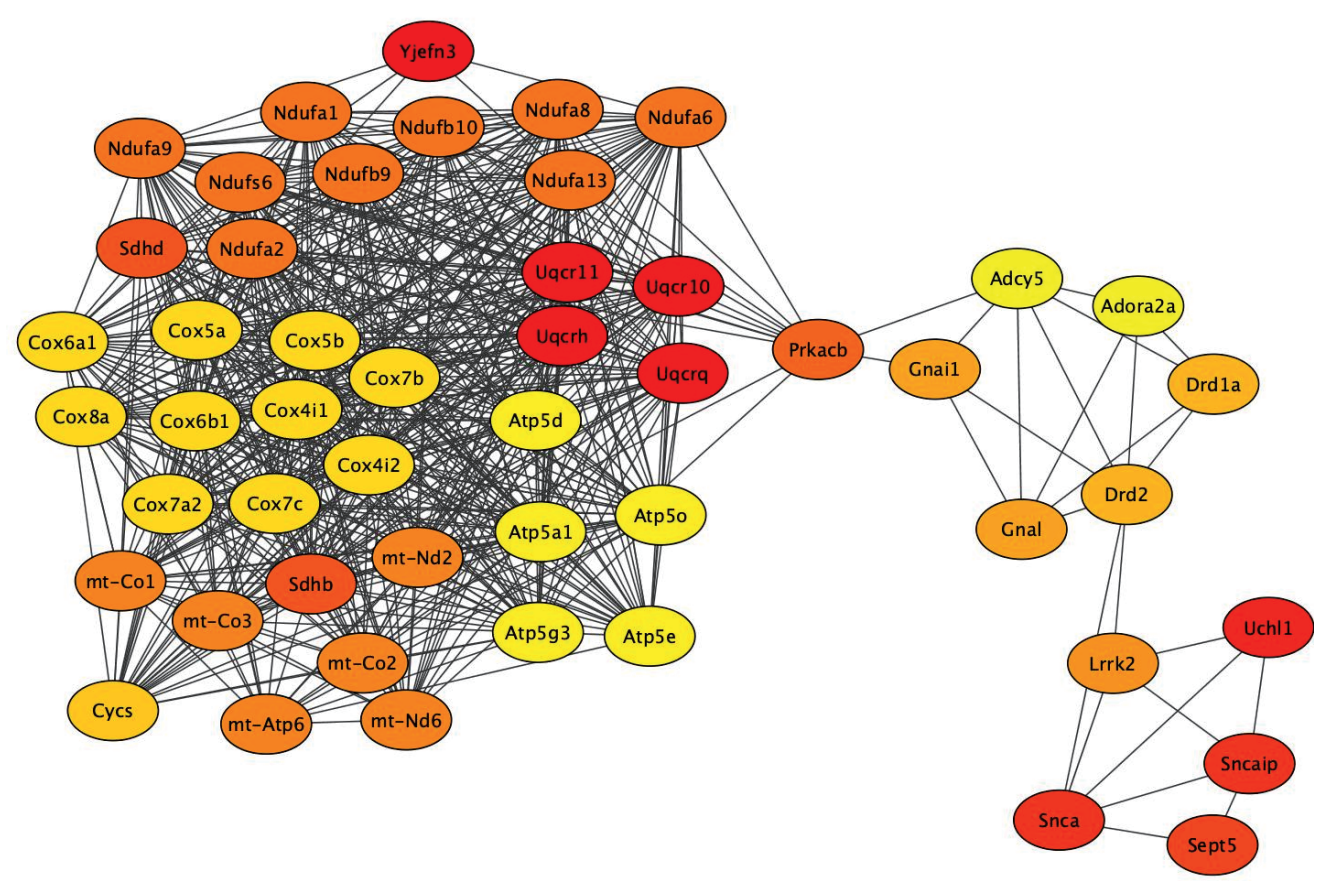

图 6 利用 STRING 数据库构建的小鼠脑纹状体相比于视网膜高表达的帕金森病通路的基因互作网络

Fig. 6 (Color online) Constructed gene network of PD pathway based on genes with higher expression in mouse striatum compared with retina

\section{3 讨 论}

帕金森病是一种复杂的多因素疾病，其致病原 因包括基因突变、年龄老化、脑外伤和环境因素 等 ${ }^{[6]}$ ，但确切的致病机理仍不清楚. 帕金森患者常 伴有视网膜的受累，后者可呈早期临床表症，可能 与多巴胺能神经元的死亡直接或间接地对视网膜产 生影响有关. 类似的大脑对视网膜的影响可能在多 种疾病中存在，例如阿尔兹海默症患者的视网膜功
能改变已经被报导，并被认为可应用于该病的早期 检测 ${ }^{[16]}$. 而根据 KHOO 等 ${ }^{[17]}$ 的研究, PD 患者独 有的 $\mathrm{VH}$ 改变存在于 $22 \%$ 的 PD 病人中, 因此视网 膜的基因表达的改变有可能作为 PD 的早期生物标 记物. 本研究通过比较转录组学和生物信息学分析 的方法，对正常小鼠的纹状体和视网膜的基因表达 谱进行比较分析，发现了两者在基因表达、功能和 通路上的异同. 同时，也揭示了 PD 通路基因在两 者中表达的情况, 对 PD 与视网膜改变关系的重要 分子基础提供参考. 


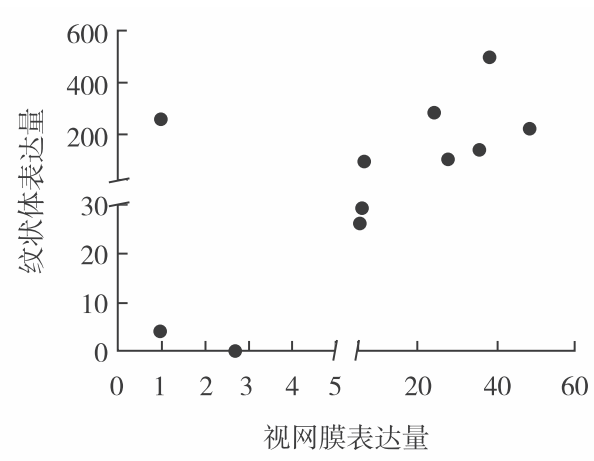

(a) $|\mathrm{lb} \mathrm{FC}|>2$ 的 12 个PD通路相关基因在两种组织间的表达

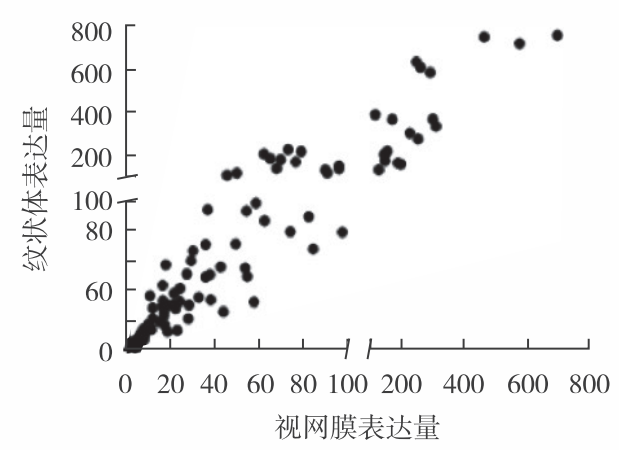

(b ) $|\mathrm{lb} \mathrm{FC}|<2$ 的 117 个PD通路相关基因在两种组织间的表达

\section{图 7 在小鼠脑纹状体和视网膜中帕金森 通路基因的差异表达比较}

Fig. 7 Comparison of differentially expressed genes of PD pathway between mouse striatum and retina

视网膜是由脑的一部分延伸发育而来. 作为视 觉器官眼的核心组成部分, 其功能上有相当程度的 特化. 本研究的转录组数据较好地验证了这一点, 发现纹状体和视网膜的转录组水平表达有明显差 异. 其中, 基因功能富集的结果显示, 在纹状体中 表现为信号转导及膜转运相关基因的高表达，这可 能是纹状体作为中枢神经系统的主要神经结构之一 的功能所决定. 而在视网膜中表现为转录和视觉感 受器相关基因的高表达, 这可能与视网膜特殊的光 感受与传导的功能有关联. 这些基因中有部分, 如 Rhodopsin 和 RP1 等, 也是遗传性视网膜疾病的重 要致病基因 ${ }^{[18-19]}$. 这说明本研究方法能较好地将特 异性表达的基因鉴定出来. 在 KEGG 通路分析中, 小鼠脑纹状体高表达的基因则富集到了一些神经疾 病的通路, 包括 PD、阿尔茨海默病和亨廷顿病等 重要的神经退行性疾病. 而视网膜的基因则显示了 剪接体等转录相关通路的富集, 这可能与视网膜高 度活跃的 RNA 加工和剪接有关 ${ }^{[20-22]}$. 在遗传性视 网膜疾病的已知致病基因中，有一部分已明确与剪 接体有关. 这也从侧面反映了所篮选的 DEG 有着
较好的代表性.

PD 疾病的信号转导通路是本研究的重点, 我 们对该通路在正常小鼠纹状体和视网膜的差异基因 表达谱情况进行了较深人的生物信息学分析, 试图 探索 PD 疾病发病的分子基础. 研究发现, 虽然纹 状体和视网膜的 PD 信号通路基因均有一定程度的 表达, 但在两种组织上有差异表达的 129 个已检测 到的基因中, 只有 12 个是在 PD 信号通路上, 不到 $10 \%$ (即 $|\mathrm{lb} \mathrm{FC}| \geqslant 2$ ), 而绝大多数 (117 个) PD 通 路基因是 $|\mathrm{lb} \mathrm{FC}|<2$ 的, 与纹状体呈现了相近的表 达水平. 这部分基因的显著改变, 提示它们可能是 PD 患者视网膜病理性改变的关键分子基础. 在 $|\mathrm{lb} \mathrm{FC}| \geqslant 2$ 的基因中, 包括了重要的 SNCA 和它的 相互作用蛋白 ( $\alpha$-synuclein-interacting protein, Sncaip), 这从一定程度上解释了为什么纹状体可能 对 Snca 的改变更加敏感. 此外, 电子传递链复合 物 Cox 家族中的 Cox4 有两个亚基, Cox4i1 及 Cox4i2, 后者是线粒体电子传递链的组成成分, 也 是 PD 通路的成分. 而在小鼠视网膜中, 仅 Cox $4 \mathrm{i} 2$ 基因的表达量高于在纹状体中的. 另外, 已知 PD 发病涉及毒素影响, 因神经毒素在体内的活性形态 $\mathrm{MPP}+$ 是鱼藤酮等环境毒素的主要攻击目标, 目前 已成为 PD 动物造模研究的金标准, 因此, 本研究 提出一个新的研究思路: MPTP 这类毒性物质诱导 的帕金森病模型可能用于 PD 视网膜的受累的分子 机制研究.

\section{结 语}

本研究利用比较转录组学和生物信息学分析, 对比分析了正常小鼠的纹状体与视网膜的基因表达 谱, 发现两者在基因表达、功能和相关信号转导通 路上存在许多明显差异, 证实了基因表达的差异在 组织器官的功能特异性方面起到重要作用. 同时, 通过对与疾病相关的分析发现, PD 信号转导通路 上的多个基因在纹状体和视网膜也有着不同程度的 表达, 存在较高程度的相似性, 可能是 PD 在纹状 体与视网膜的共同分子发病基础, 可为进一步研究 PD 与视网膜的病变关系提供参考.

基金项目: 国家自然科学基金资助项目（31671311）

作者简介: 须周恒 $(1991-)$, 江南大学硕士研究生. 研究方向: 全转录组研究在神经系统疾病中的应用. E-mail: zhx_bio@ hotmail.com

引文: 须周恒, 朱营利, 孙孟菲, 等. 小鼠纹状体与视网膜的 比较转录组学分析 $[\mathrm{J}]$. 深圳大学学报理工版, 2020,37 (1) : $17-24$ 


\section{参考文献 / References :}

[ 1 ] KALIA L V, LANG A E. Parkinson's disease [J]. Lancet, 2015, 386(9996) : 896-912.

[ 2 ] OERTEL F C, ZIMMERMANN H G, BRANDT A U, et al. Novel uses of retinal imaging with optical coherence tomography in multiple sclerosis $[\mathrm{J}]$. Expert Review of Neurotherapeutics, 2018, 27 : 1-13.

[ 3 ] CRISCUOLO C, CERRI E, FABIANI C, et al. The retina as a window to early dysfunctions of Alzheimer's disease following studies with a $5 \times$ FAD mouse model $[\mathrm{J}]$. Neurobiology of Aging, 2018, 67: 181-188.

[ 4 ] PAIVA F T M, SILVERSTEIN S M, BUTLER P D, et al. Color vision impairments in schizophrenia and the role of antipsychotic medication type $[\mathrm{J}]$. Schizophr Research, 2018, S0920-9964(18) : 30551-30556.

[ 5 ] LEE J Y, AHN J, KIM T W, et al. Optical coherence tomography in Parkinson's disease: is the retina a biomarker? [J]. Journal of Parkinsons Disease, 2014, 4 (2) : 197-204

[ 6 ] RAMIREZ A I, De HOZ R, SALOBRAR-GARCIA E, et al. The role of microglia in retinal neurodegeneration: Alzheimer's disease, Parkinson, and glaucoma [ J ] . Frontiers in Aging Neuroscience, 2017, 9: 214-234.

[ 7 ] de MAINDREVILLE A D, FÉNELON G, MAHIEUX F. Hallucinations in Parkinson's disease: a follow-up study [J]. Movement Disorders, 2005, 20(2) : 212-217.

[ 8 ] ONOFRJ M, BONANNI L, ALBANI G, et al. Visual hallucinations in Parkinson's disease: clues to separate origins $[\mathrm{J}]$. Journal of the Neurological Sciences, 2006, $248(1 / 2): 143-150$.

[ 9 ] SONG Fuhai, QIAN Ying, PENG Xing, et al. Perturbation of the transcriptome: implications of the innate immune system in Alzheimer's disease $[\mathrm{J}]$. Current Opinion in Pharmacology, 2016, 26: 47-53.

[10 ] ANNIBALI V, UMETON R, PALERMO A, et al. Analysis of coding and non-coding transcriptome of peripheral $\mathrm{B}$ cells reveals an altered interferon response factor (IRF)-1 pathway in multiple sclerosis patients $[\mathrm{J}]$. Journal of Neuroimmunology, 2018, 324: 165-171.

[11] ENWRIGHT III J F, HUO Zhiguang, ARION D, et al. Transcriptome alterations of prefrontal cortical parvalbumin neurons in Schizophrenia $[\mathrm{J}]$. Molecular psychiatry, 2018, 23(7) : 1606-1613.

[12] SANDOR C, ROBERTSON P, LANG C, et al. Transcriptomic profiling of purified patient-derived dopamine neurons identifies convergent perturbations and therapeutics for Parkinson's disease [ J ]. Human Molecular Genetics, 2017, 26(3) : 552-566.

[13] GANESHAN V, SKLADNEV N V, KIM J Y, et al. Preconditioning with remote photobiomodulation modulates the brain transcriptome and protects against MPTP insult in mice $[\mathrm{J}]$. Neuroscience, 2019, 400: 85-97.
[14] HUANG Dawei, SHERMAN B T, LEMPICKI $R$ A. Systematic and integrative analysis of large gene lists using DAVID bioinformatics resources $[\mathrm{J}]$. Nature Protocols, 2009: 4(1): 44-57.

[15] HUANG Dawei, SHERMAN B T, LEMPICKI $\mathrm{R}$ A. Bioinformatics enrichment tools: paths toward the comprehensive functional analysis of large gene lists $[\mathrm{J}]$. Nucleic Acids Research, 2009: 37(1) : 1-13.

[16] SHARIFLOU S, GEORGEVSKY D, MANSOUR H, et al. Diagnostic and prognostic potential of retinal biomarkers in early on-set Alzheimer's disease [J]. Current Alzheimer Research, 2017, 14(9): 1000-1007.

[17 ] KHOO T K, YARNALL A J, DUNCAN G W, et al. The spectrum of nonmotor symptoms in early Parkinson disease [J]. Neurology, 2013, 80(3) : 276-281.

[18] ATHANASIOU D, AGUILÀ M, BELLINGHAM J, et al. The molecular and cellular basis of rhodopsin retinitis pigmentosa reveals potential strategies for therapy $[\mathrm{J}]$. Progress in Retin Eye Reserach, 2017, 62: 1-23.

[19] JACOBSON S G, CIDECIYAN A V, IANNACCONE A, et al. Disease expression of RP1 mutations causing autosomal dominant retinitis pigmentosa $[\mathrm{J}]$. Investigative Ophthalmology and Visual Science, 2000, 41(7) : 1898-1908.

[20 ] BERGER A, LORAIN S, JOSEPhine C, et al. Repair of rhodopsin mRNA by spliceosome-mediated RNA transsplicing: a new approach for autosomal dominant retinitis pigmentosa [J]. Molecular Therapy, 2015, 23(5) : 918930.

[21] XU Mingchu, XIE Yajing, ABOUZEID H, et al. Mutations in the spliceosome component, $\mathrm{CWC} 27$, cause retinal degeneration with or without additional developmental anomalies $[\mathrm{J}]$. American Journal of Human Genetics, 2017, 100 (4) : 592-604.

[22] TANACKOVIC G, RANSIJN A, THIBAULT P, et al. PRPF mutations are associated with generalized defects in spliceosome formation and pre-mRNA splicing in patients with retinitis pigmentosa $[\mathrm{J}]$. Human Molecular Genetics, 2011, 20(11) : 2116-2130.

[23] SIDDIQUI I J, PERVAIZ N, ABBASI A A. The Parkinson disease gene SNCA: evolutionary and structural insights with pathological implication $[\mathrm{J}]$. Scientific Reports, 2016, 6(1) : 24475-24485.

【中文责编：英 子; 英文责编：艾 琳】 